\title{
COBERTURA NITROGENADA EM GIRASSOL SOB PLANTIO DIRETO NA PALHA: TEORES DE NUTRIENTES NAS FOLHAS ${ }^{1}$
}

\section{Broadcasting Nitrogen In No-Tillage Of Sunflower: Foliate Nutrient Content}

\author{
Denise Bruginski de Carvalho² \\ Amir Pissaia ${ }^{3}$
}

\section{Resumo}

O presente trabalho teve como objetivo avaliar o efeito de doses de N (nitrogênio), aplicadas em cobertura sobre o teor de nutrientes nas folhas de girassol (Helianthus annuus L.), em sistema de plantio direto. O experimento foi realizado na Fazenda Agripastos, em Palmeira, Estado do Paraná, em Latossolo VermelhoAmarelo, cultivado em sistema de plantio direto desde 1976. Foram avaliadas as doses 0 (testemunha), 25 , $50,75,100$ e $125 \mathrm{~kg}$ de $\mathrm{N} \mathrm{ha}^{-1}$ (uréia), aplicadas em cobertura no estádio $\mathrm{V}_{8}$. Os teores foliares de $\mathrm{N}, \mathrm{P}, \mathrm{K}$, $\mathrm{Ca}, \mathrm{Mg}, \mathrm{Fe}, \mathrm{Mn}$, Cu e Zn foram determinados no estádio $\mathrm{R}_{4}$. Foram avaliados: número de plantas acamadas, quebradas e com sintomas de podridão do capítulo (Sclerotinia sclerotiorum). A relação entre os teores de $\mathrm{N}$ nas folhas (y) e as doses de $\mathrm{N}$ aplicadas ( $\mathrm{x}$ ) foi linear ( $\mathrm{y}=44,4+0,07 \mathrm{x}, \mathrm{R}^{2}=0,87$ ). Não foram evidenciados sintomas de excesso, mesmo nas maiores doses de N. O N aplicado em cobertura alterou o seu teor nas folhas, mas não alterou os teores de $\mathrm{P}, \mathrm{K}, \mathrm{Ca}, \mathrm{Mg}$, Fe, Mn, Cu e Zn.

Palavras-chave: Helianthus annuus; Nutrição mineral; Sistemas de produção.

\section{Abstract}

The present research was done in order to evaluate the effect of covering fertilization with different $\mathrm{N}$ doses on foliar nutrient content of sunflower (Helianthus annuus L.) under no-tillage cropping system. The experiment was conducted at Fazenda Agripastos, in Palmeira, state of Paraná, in a red-yellow latossol, cultivated under no-tillage system since 1976. Nitrogen doses of 0 (witness), 25, 50, 75, 100 and $125 \mathrm{~kg}$ $\mathrm{ha}^{-1}$ (urea), were applied in covering, at $\mathrm{V}_{8}$ sunflower fenological stage. Foliar analysis were done at $\mathrm{R}_{4}$ stage in order to measure $\mathrm{N}, \mathrm{P}, \mathrm{K}, \mathrm{Ca}, \mathrm{Mg}, \mathrm{Fe}, \mathrm{Mn}, \mathrm{Cu}$ and $\mathrm{Zn}$ contents. The number of laying and broken plants, and plant with head rot symptoms (Sclerotinia sclerotiorum) were also evaluated. The relation between foliar $\mathrm{N}$ content ( $\mathrm{y}$ ) and $\mathrm{N}$ doses applied $(\mathrm{x})$ was linear $\left(\mathrm{y}=44,4+0,07 \mathrm{x}, \mathrm{R}^{2}=0,87\right)$. It was not proven excess symptoms, even at the largest doses of $\mathrm{N}$. The broadcasting nitrogen altered $\mathrm{N}$ content of sunflower leaves but did not influence $\mathrm{P}, \mathrm{K}, \mathrm{Ca}, \mathrm{Mg}, \mathrm{Fe}, \mathrm{Mn}, \mathrm{Cu}$ and $\mathrm{Zn}$ contents.

Keywords: Helianthus annuus; Mineral nutrition; Production system.

1 Extraído da dissertação de mestrado do primeiro autor, apresentada ao Curso de Pós-graduação em Agronomia - Produção Vegetal, Universidade Federal do Paraná.

2 Engenheira Agrônoma, CREA 28300-D, M.Sc., aluna de doutorado do Curso de Pós-graduação em Agronomia - Produção Vegetal, UFPR, bolsista da CAPES. Rua dos Funcionários, 1540, Curitiba/PR, CEP 80035-050. denisehb@agrarias.ufpr.br

3 Engenheiro Agrônomo, CREA/PR 3480-D, Dr., professor adjunto do Departamento de Fitotecnia e Fitossanitarismo, Setor de Ciências Agrárias, UFPR. 


\section{Introdução}

A planta de girassol absorve maior quantidade de macronutrientes em comparação com outras culturas de grãos como a soja, o milho e o trigo (ROSSI, 1991). Durante o período vegetativo, o ritmo de absorção de $\mathrm{N}$ é mais rápido do que no período reprodutivo, sendo de grande importância para o girassol encontrá-lo em uma forma facilmente assimilável, para que possa se acumular nos tecidos jovens (SFREDO et al., 1984; VRÂNCEANU, 1977). O período onde ocorre maior taxa de absorção de nutrientes e crescimento mais acelerado do girassol é o da fase imediatamente após a formação do botão floral até o final do florescimento (CASTRO et al., 1997).

Segundo Bayer e Scheneider (1999), áreas cultivadas sob sistema de plantio direto apresentam, nos primeiros anos, um processo de acúmulo de matéria orgânica, a qual tende a reduzir gradualmente com o passar do tempo, até atingir um conteúdo estável, que representa um acréscimo considerável quando comparado ao teor existente antes da implantação do sistema (MUZILLI, 1985). Conseqüentemente, ocorre aumento nos teores de C orgânico total e na capacidade de troca catiônica do solo (BAYER; SCHENEIDER, 1999), resultando em efeitos diferenciados na nutrição de plantas (MUZILLI, 1985).

Uma fertilização excessiva com $\mathrm{N}$ deve ser evitada, pois além do desperdício de fertilizante, provoca crescimento acentuado da parte aérea, desenvolvimento inadequado dos tecidos de sustentação e susceptibilidade ao ataque de fungos e insetos (LARCHER, 2000; LEITE, 1997; ROSSI, 1998). A presença de tecidos suculentos aumenta a pro- babilidade de acamamento, quebra do caule e aparecimento de doenças (MALAVOLTA et al., 1997).

A planta de girassol é hospedeira de mais de 35 microrganismos fitopatogênicos, a maioria fungos, que podem levar à redução qualitativa e quantitativa do produto (CARTER, 1978). A podridão branca da haste e do capítulo, causada pelo fungo Sclerotinia sclerotiorum (Lib.) de Bary é considerada a doença que requer maior atenção no cultivo do girassol. O fungo persiste durante muitos anos no solo, podendo ocasionar podridão basal, podridão da haste ou podridão do capítulo (LEITE, 1997).

O objetivo deste trabalho foi avaliar o efeito de doses de $\mathrm{N}$ aplicadas em cobertura no cultivo de girassol, em sistema de plantio direto, nos teores foliares de macro e micronutrientes e sobre a susceptibilidade a Sclerotinia sclerotiorum (Lib.) de Bary.

\section{Materiais e métodos}

O trabalho foi conduzido na Fazenda Agripastos, no município de Palmeira, região dos Campos Gerais do Paraná, localizado nas coordenadas de $25^{\circ} 21^{\prime}$ de latitude Sul e $49^{\circ} 56^{\prime}$ de longitude Oeste e altitude de 820 m (FUNDAÇÃO INSTITUTO AGRONÔMICO DO PARANÁ, 1984), em 1998/1999, em solo classificado como podzólico vermelho-amarelo, textura média, fase campo subtropical e relevo suave ondulado (EMBRAPA/CNPS, 1999), cujas características químicas são apresentadaos na Tabela 1. 


\section{Tabela 1 - Caracterização química do solo da área experimental, Fazenda Agripastos, Pal- meira, PR. 1998. ${ }^{1}$}

Table 1 - Soil chemical characterization of the experimental area, Agripastos Farm, Palmeira, PR. 1998. ${ }^{1}$

\begin{tabular}{ccccccccc}
\hline $\begin{array}{c}\text { Profundida } \\
\text { de } \\
(\mathrm{cm})\end{array}$ & $\mathrm{pH}$ & $\mathrm{Al}^{+3}$ & $\mathrm{H}+\mathrm{Al}$ & $\begin{array}{c}\mathrm{Ca}^{+2}+ \\
\mathrm{Mg}^{+2}\end{array}$ & $\mathrm{~K}^{+}$ & $\mathrm{P}$ & $\begin{array}{c}\text { Matéria } \\
\text { orgânica }\end{array}$ & $\begin{array}{c}\text { Saturação } \\
\text { por bases }\end{array}$ \\
\hline $\mathrm{CaCl}$ & & & $\left(\mathrm{Cmol}_{\mathrm{c}} \mathrm{dm}^{-3}\right)$ & & $\left(\mathrm{mg} \mathrm{dm}^{-3}\right)$ & $\mathrm{g} \mathrm{kg}^{-1}$ & $\%$ \\
$10-20$ & 4,4 & 0,3 & 5,8 & 4,2 & 0,2 & 6,0 & 33 & 43,1 \\
$20-40$ & 4,2 & 0,5 & 6,2 & 3,0 & 0,1 & 1,0 & 30 & 33,5 \\
\hline
\end{tabular}

${ }^{1}$ Análises realizadas no Laboratório de Análises de Solos do Departamento de Solos da UFPR.

A área experimental é explorada para cultivo agrícola desde 1959, sendo que o sistema de plantio direto foi adotado em 1976. A rotação de culturas feita desde 1981, intercala, nas safras de inverno, os cultivos de aveia-preta, cevada e trigo e no verão, soja e milho, que neste experimento foi substituído pelo girassol.

Foi utilizado o delineamento de blocos ao acaso com quatro repetições. Os tratamentos constituíram-se de seis dosagens: 0, 25, 50, 75, 100

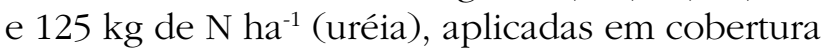
no estádio $\mathrm{V}_{8}$ conforme a descrição feita por Schneiter e Miller (1981), também apresentada por Castiglioni et al. (1994). A aplicação foi realizada 25 dias após a emergência, segundo a recomendação da Embrapa (CASTRO et al., 1997). As parcelas (seis linhas com dez metros de comprimento), perfizeram área total de $54 \mathrm{~m}^{2}$, com média de 4,5 plantas $\mathrm{m}^{-2}$.

Antes da semeadura da cultura de inverno, foram aplicados $1.500 \mathrm{~kg} \mathrm{ha}^{-1}$ de calcário dolomítico (PRNT= 76\%), distribuído a lanço em superfície. Na semeadura a saturação de bases era de $43,1 \%$ na camada de $0-10 \mathrm{~cm}$ de profundidade (Tabela 1).

O cultivo do girassol foi feito em sistema de plantio direto, sobre cobertura morta, formada pelo cultivo da aveia-preta (Avena strigosa), dessecada uma semana antes da semeadura, por meio da aplicação de $960 \mathrm{~g} \mathrm{ha}^{-1}$ de glyphosate, misturado com 0,5 $1 \mathrm{ha}^{-1}$ de óleo mineral. A quantidade de matéria seca formada pela aveia-preta foi estimada em $3.400 \mathrm{~kg} \mathrm{ha}^{-1}$.

Foi utilizada uma semeadora de grãos, PAR 2800, apropriada para o sistema de plantio direto, com quatro linhas de semeadura distanciadas a $0,90 \mathrm{~m}$ e discos recomendados para o cultivo do girassol, regulados para a distribuição de cinco sementes por metro de sulco, visando ao estabelecimento de uma população de 50 mil plantas ha' ${ }^{-1}$, considerando o poder de germinação das sementes como foi sugerido por Castro et al. (1997).

Segundo Castro et al. (1997), na semeadura convencional em sulcos, realizada em setembro de 1998, conforme a recomendação, foram aplicados $300 \mathrm{~kg} \mathrm{ha}^{-1}$ da fórmula 00-26-26 junto com as sementes do híbrido M 734, conforme prática recomendada no Estado do Paraná (EMBRAPA/CNPSo, 1997). Esse híbrido apresenta ciclo tardio, com maturação fisiológica aos 103 dias após a emergência e teor de óleo nos aquênios de aproximadamente 41\% (ROSSI, 1998).

Dois dias antes da aplicação dos tratamentos, foi feito o sulcamento a $8 \mathrm{~cm}$ de profundidade e $20 \mathrm{~cm}$ de distância das linhas de cultivo, utilizando uma adubadora adaptada para aplicação localizada de $\mathrm{N}$ em cobertura, no cultivo do milho em sistema de plantio direto.

A quantidade de uréia, correspondente aos respectivos tratamentos em cada linha de plantio, foi distribuída quando as plantas se encontravam no estádio de desenvolvimento $\mathrm{V}_{8}$ e imediatamente incorporada, com auxílio de enxada.

Segundo Castro et al. (1997), foi realizada aplicação de boro, conforme recomendação da Embrapa, na dose de $1,0 \mathrm{~kg}$ de $\mathrm{B} \mathrm{ha}^{-1}$ (bórax) dissolvido previamente em água a $70^{\circ} \mathrm{C}$ e aplicado com pulverizador costal manual, em $300 \mathrm{~L} \mathrm{ha}^{-1}$ de calda, quando as plantas se encontravam no estádio de desenvolvimento $\mathrm{V}_{9}$. 
Para a determinação do N, P, K, Ca, Mg, $\mathrm{Fe}, \mathrm{Mn}, \mathrm{Cu}$ e $\mathrm{Zn}$ foram coletadas folhas do terço superior de cinco plantas por parcela no estágio $\mathrm{R}_{4}$, conforme recomendação (MALAVOLTA et al., 1997). As análises foram realizadas no Laboratório de Nutrição Mineral de Plantas do Departamento de Solos da Universidade Federal do Paraná, utilizando-se o método Kjeldahl para digestão de N total e para os demais elementos a digestão com $\mathrm{HCl}(0,3 \mathrm{~N})$, conforme Hildebrand (1977).

Por ocasião da colheita, em fevereiro de 1999, quando as plantas estavam no estádio de maturação de colheita, foram realizadas a contagem do número de plantas com ângulo de inclinação maior que $45^{\circ}$ em relação à vertical, para avaliação do acamamento; a contagem do número de plantas quebradas; e a contagem do número de plantas infectadas por Sclerotinia sclerotiorum (Lib) de Bary, apresentando sintomas de podridão do capítulo.

Os resultados foram submetidos à análise de variância utilizando o programa MSTATC. As variâncias dos tratamentos foram analisadas quanto à homogeneidade, pelo teste de Bartlett. As médias dos tratamentos foram testadas pelo teste F. Quando os resultados revelaram diferenças estatisticamente significantes entre as médias de tratamentos, foram ajustadas pelo método de regressão linear, como sugere Koehler (1998).

\section{Resultados}

A relação entre os teores de $\mathrm{N}$ nas folhas (y) e as doses de $\mathrm{N}$ aplicadas ( $\mathrm{x}$ ) foi linear ( $\mathrm{y}=$ $\left.44,4+0,07 \mathrm{x}, \mathrm{R}^{2}=0,87\right)$. Sem aplicação de $\mathrm{N}$, o seu teor na folha foi de $44,4 \mathrm{~g} \mathrm{~kg}^{-1} \mathrm{e}$ com a dose máxima (125 kg de $\mathrm{N} \mathrm{ha}^{-1}$ ) foi de $53,1 \mathrm{~g} \mathrm{~kg}^{-1}$.

O N participa na fixação biológica, redução e assimilação do nitrato, na síntese de proteínas e ácidos nucléicos, além de constituinte de aminoácidos, proteínas, enzimas, coenzimas, ácidos nucléicos, bases nitrogenadas, vitaminas, glico e lipoproteínas, pigmentos e outros produtos secundários. Participa, também, nos processos de absorção iônica, fotossíntese, respiração, sínteses, multiplicação, diferenciação celular e herança genética $(16 ; 17)$. Para fins de diagnose do estado nutricional do girassol, Malavolta et al., (1997) consideram ótimo o intervalo entre 33 a $35 \mathrm{~g}$ de $\mathrm{N} \mathrm{kg}$ 1. Deibert e Utter (1989) comparando níveis de nutrientes nas folhas de girassol sob diferentes sistemas de cultivo, encontraram valores entre $16 \mathrm{e}$ $27 \mathrm{~g}_{\text {de }} \mathrm{N} \mathrm{kg}^{-1} \mathrm{e}$, comparando com outros dados de literatura, consideraram esta faixa como dentro da normalidade.

No presente trabalho, em todos os tratamentos foram encontrados valores de $\mathrm{N}$ superiores aos citados por outros autores, o que revela a existência desse nutriente disponível inclusive nas parcelas testemunhas. O plano de rotação de culturas e o cultivo sob sistema de plantio direto desde 1976 podem ter facilitado a condição de equilíbrio na disponibilidade de $\mathrm{N}$ proveniente da mineralização da matéria orgânica, presente em grande quantidade no solo (33 $\mathrm{g} \mathrm{kg}^{-1}$ na profundidade de 0 a $10 \mathrm{~cm}$ ).

Segundo Sameni et al. (1976), o teor foliar de $\mathrm{N}$ está diretamente relacionado com o seu teor total no solo, com a sua disponibilidade através da mineralização da matéria orgânica do solo e com a quantidade deste elemento fornecido por meio da adubação. Logo, a adubação nitrogenada em cobertura teve influência sobre o teor foliar de $\mathrm{N}$ e a diferença foi constatada por meio da análise estatística dos dados.

O histórico da área experimental e os resultados encontrados sugerem que a quantidade mínima de $\mathrm{N}$ necessária para o desenvolvimento do girassol já estava presente no solo, e que a adubação realizada foi além da necessidade do girassol.

Complementarmente, Larcher (2000) afirma que uma fertilização excessiva com $\mathrm{N}$ pode provocar desequilíbrios no crescimento e desenvolvimento da planta, diminuindo sua tolerância aos estresses climáticos e aumentando sua susceptibilidade ao ataque de fungos.

Entretanto, não houve diferença significativa entre os tratamentos para a presença de plantas acamadas, quebradas e com sintomas de podridão do capítulo (Tabela 2). 
Tabela 2 - Percentual médio de plantas acamadas, quebradas e com sintomas de podridão do capítulo submetido a seis doses de nitrogênio em cobertura, em sistema de plantio direto. Palmeira, PR. 1998/99.

Table 2 - Average percentage of sick plants, broken and with flower-head rottenness symptoms, submitted to six doses of nitrogen on covering, in direct plantation system. Palmeira, PR. 1998/99.

\begin{tabular}{cccc}
\hline $\mathbf{N}\left(\mathbf{k g ~ h a ́}^{-1}\right)$ & Plantas acamadas & Plantas quebradas & $\begin{array}{c}\text { Plantas com sintoma de } \\
\text { podridão do capítulo }\end{array}$ \\
\hline $\mathbf{0}$ & 1,4 & 0,5 & 0,7 \\
$\mathbf{2 5}$ & 1,5 & 0,0 & 0,5 \\
$\mathbf{5 0}$ & 1,2 & 0,6 & 0,6 \\
$\mathbf{7 5}$ & 2,3 & 0,6 & 0,8 \\
$\mathbf{1 0 0}$ & 2,0 & 0,7 & 0,2 \\
$\mathbf{1 2 5}$ & 2,0 & 0,2 & 0,6 \\
\hline Média & 1,7 & 0,4 & 0,6 \\
\hline C.V.(\%) & 13,18 & 6,35 & 16,96 \\
\hline
\end{tabular}

A análise de variância dos dados revelou não haver diferença significativa entre os tratamentos. A quantidade de plantas acamadas e quebradas não evidenciou efeito da adubação nitrogenada, pois os valores percentuais encontrados não representam perdas significativas para o cultivo do girassol.

A contagem de plantas com sintomas de podridão do capítulo, causada pelo fungo Sclerotinia sclerotiorum (Lib.) de Bary, revelou a existência de $0,6 \%$ de incidência média na área do experimento, o que significa uma infestação de cerca de 300 plantas ha ${ }^{-1}$. Mesmo não havendo aumento da incidência relacionada às quantidades de $\mathrm{N}$ aplicado, este resultado encontrado ressalta a importância da rotação de culturas neste sistema de cultivo, em solo com elevado teor de matéria orgânica, já que a forma de sobrevivência do fungo (escleródio) multiplicada muitas vezes devido ao tamanho dos capítulos, pode se tornar fonte de inóculo para culturas sensíveis, como o caso da soja.

Entretanto, não foi comprovado pela análise estatística dos dados nenhum sintoma de excesso, mesmo nas maiores doses de $\mathrm{N}$.

Marschner (1993), Mengel e Kirkby (1987) afirmam que o excesso de $\mathrm{N}$ também pode induzir à deficiência de outros nutrientes, como o $\mathrm{P}$ e K. Todavia, os teores foliares de P, K, Ca e Mg não apresentaram diferenças significativas entre os tratamentos (Tabela 3 e 4).

\section{Tabela 3 - Teores médios de macronutrientes nas folhas de girassol submetido a seis doses} de nitrogênio em cobertura, em sistema de plantio direto. Palmeira, PR. 1998/99.

Table 3 - Average contents of macronutrients in leaves of sunflower submitted to six doses of nitrogen on covering, in direct plantation system. Palmeira, PR. 1998/99.

\begin{tabular}{ccccc}
\hline \multirow{2}{*}{$\mathbf{N}\left(\mathbf{k g ~ h a}^{-1}\right)$} & $\mathrm{P}$ & $\mathrm{K}$ & $\mathrm{Ca}$ & $\mathrm{Mg}$ \\
\cline { 2 - 5 } & 4,3 & 37,0 & 17,6 & 4,8 \\
25 & 5,0 & 36,3 & 16,7 & 5,0 \\
50 & 4,4 & 35,0 & 17,0 & 4,8 \\
75 & 5,1 & 36,8 & 16,5 & 4,7 \\
100 & 5,5 & 36,7 & 16,1 & 4,7 \\
125 & 5,3 & 34,5 & 15,6 & 4,6 \\
\hline Média & 4,9 & 36,1 & 16,6 & 4,8 \\
\hline C.V. $(\%)$ & 20,65 & 9,37 & 6,96 & 9,29 \\
\hline
\end{tabular}


Tabela 4 - Teores médios de micronutrientes nas folhas de girassol submetido a seis doses de nitrogênio em cobertura, em sistema de plantio direto. Palmeira, PR. 1998/99.

Table 4 - Average contents of micronutrients in leaves of sunflower submitted to six doses of nitrogen on covering, in direct plantation system, Palmeira, PR, 1998/99.

\begin{tabular}{ccccc}
\hline & \multicolumn{4}{c}{ Micronutrientes $\left(\mathrm{mg} \mathrm{kg}^{-1}\right)$} \\
\cline { 2 - 5 } & $\mathrm{Fe}$ & $\mathrm{Mn}$ & $\mathrm{Cu}$ & $\mathrm{Zn}$ \\
\hline 0 & 140,5 & 135,3 & 25,8 & 38,3 \\
25 & 155,0 & 146,5 & 23,8 & 40,3 \\
50 & 139,3 & 107,3 & 24,8 & 41,8 \\
75 & 140,0 & 99,0 & 27,0 & 44,3 \\
100 & 142,5 & 108,0 & 27,5 & 45,5 \\
125 & 139,3 & 115,3 & 27,5 & 44,8 \\
\hline Média & 142,7 & 118,6 & 26,1 & 72,5 \\
\hline C.V.(\%) & 14,34 & 29,22 & 10,25 & 11,39
\end{tabular}

O teor de $\mathrm{P}$ pode ser considerado adequado, se comparado com a faixa de 4 a 7 g de $\mathrm{P}$ $\mathrm{kg}^{-1}$ indicada por Malavolta etal. (1997), para diagnose do estado nutricional de girassol. Deibert e Utter (1989) registraram valores entre 2,2 e 5,2 g de $\mathrm{P} \mathrm{kg}^{-1}$ como sendo adequados para folhas de girassol no início de florescimento. De acordo com Tomé (1997), o teor de P, antes da instalação do experimento, nas camadas de 0-10 cm é considerado médio e baixo na camada de $10-40 \mathrm{~cm}$ (Tabela 1), considerando a textura média do solo. Contudo, em função dos baixos teores deste elemento no solo, não foi constatada interferência negativa dos teores de $\mathrm{N}$ sobre os teores de $\mathrm{P}$, nem mesmo no seu conteúdo foliar.

O teor foliar de $\mathrm{K}\left(36,1 \mathrm{~g} \mathrm{~kg}^{-1}\right)$ apresentou-se acima da faixa ideal (20 a $24 \mathrm{~g} \mathrm{~kg}^{-1}$ ) citada por Malavolta et al. (1997). Mengel e Kirkby (1987) consideram que o K pode estar preso aos grupos carboxílicos e fenólicos da matéria orgânica, na forma trocável, e também no seu interior, sendo liberado pelo processo de mineralização. Desta forma, o elevado teor de matéria orgânica do solo (33 $\left.\mathrm{g} \mathrm{kg}^{-1}\right)$, na camada de $0-10 \mathrm{~cm}$ (Tabela 1), pode estar relacionado com os altos teores de $\mathrm{K}$ nas folhas. Borkert et al. (1997) afirmaram que o nível crítico de K para o girassol é de $18,8 \mathrm{~g} \mathrm{~kg}^{-1}$, o que difere do resultado obtido neste experimento.

O teor médio de Ca encontrado nas folhas de girassol (16,6 $\left.\mathrm{g} \mathrm{kg}^{-1}\right)$ está próximo do ideal, que se situa entre 17 e $22 \mathrm{~g} \mathrm{~kg}^{-1}$, segundo Malavolta et al. (1997). O Ca é um elemento praticamente imóvel na planta. É essencial na estrutura das células, na forma de pectatos de Ca integran- tes da lamela média da parede celular, mantém a estrutura da membrana celular, uni os fosfolipídios e garante seletividade (SALISBURY; ROSS, 1992). Marschner (1993) considera que o excesso de N pode induzir sintomas de deficiência de Ca. Além do excesso de N, o pH baixo também pode reduzir a disponibilidade de $\mathrm{K}$, Ca e Mg, como foi discutido por Malavolta etal. (1997). No caso do solo onde foi realizado o presente experimento, o pH baixo (Tabela 1) apresenta-se como um dos fatores responsáveis pelos valores de Ca encontrados na folhas de girassol.

O teor de Mg está abaixo da faixa normal, da ordem de 9 a $11 \mathrm{~g} \mathrm{~kg}^{-1}$, para folhas de girassol, considerando os valores de referência sugeridos por Malavolta et al. (1997), para fins de diagnose do estado nutricional da planta. Além da condição de pH do solo não ser ideal para a absorção de Mg, existe a possibilidade de deficiência ocasionada pela competição com outros cátions, como o K, como já foi mencionado por Mengel e Kirkby (1987). Apesar dos teores de K no solo serem considerados de médios a baixos pelos valores de referência propostos por Tomé (1997), a absorção resultou em teores foliares acima da faixa ideal.

Não houve diferença significativa entre os tratamentos em relação aos teores de $\mathrm{Fe}, \mathrm{Mn}$, Cu e Zn (Tabela 4). Sfredo e Sarruge (1990a) afirmam que o Fe é o micronutriente mais absorvido pelo girassol. Mesmo estando presente em maior quantidade que os demais micronutrientes estudados, o teor de Fe pode ser considerado um pouco abaixo da faixa de 150 a $300 \mathrm{mg} \mathrm{kg}^{-1}$, considerada por 
Malavolta etal. (1997) ideal para folhas de girassol em bom estado nutricional. A relação existente entre $\mathrm{K} / \mathrm{Ca}$ maior que dois, resultante de uma maior quantidade de K e elevado teor de ácidos orgânicos no solo, pode estar relacionada com a redução dos teores foliares de Fe, conforme observações feitas por Marschner (1993).

Quanto ao $\mathrm{Cu}$, a faixa considerada adequada por Malavolta et al. (1997) é de 30 a 50 mg kg-1 e a média encontrada neste experimento de 26,1 $\mathrm{mg} \mathrm{kg}^{-1}$ poderia ser considerada baixa. Porém, o intervalo de concentração considerado suficiente por Sfredo e Sarruge (1990b) é de 28 a $30 \mathrm{mg} \mathrm{kg}^{-1}$ e por Machado (1979) é de 21 a $23 \mathrm{mg}$ $\mathrm{kg}^{-1}$, o que é bastante variável, indicando que não se pode estabelecer uma conclusão definitiva, quando são referenciados esses autores.

Segundo Mengel e Kirkby (1987), a quantidade de matéria orgânica presente no solo interfere na disponibilidade de Mn, pela possível formação de complexos insolúveis. O teor médio encontrado nas folhas de $118,6 \mathrm{mg} \mathrm{kg}^{-1}$ está bem abaixo do limite de 300 a $600 \mathrm{mg} \mathrm{kg}^{-1}$ considerado adequado por Malavolta et al. (1997), para o bom estado nutricional do girassol. Entretanto, Sfredo e Sarruge (1990b) sugerem 180 a 220 mg de Mn kg${ }^{1}$ como referencial de normalidade para plantas de girassol, Machado (1979) relaciona valores de 101 a $250 \mathrm{mg} \mathrm{kg}^{-1}$, como adequados.

Sfredo e Sarruge (1990b) concluíram que para fins de diagnose foliar de girassol em início de floração, a concentração de $36 \mathrm{mg}$ de $\mathrm{Zn} \mathrm{kg}^{-1}$ é suficiente. Contudo, o teor de $\mathrm{Zn}$ encontrado foi de $72,5 \mathrm{mg} \mathrm{kg}^{-1}$ e está dentro da faixa de 70 e 140 mg kg-1 (15) comum em plantas bem nutridas, segundo Malavolta et al. (1997).

Larcher (2000) concluiu que as condições químicas da rizosfera e os ajustes metabólicos que a planta deve realizar em relação as mesmas condições determinam a oferta de minerais para o vegetal. Apesar das considerações feitas a respeito das condições nutricionais do girassol, as plantas não apresentaram sintomas de deficiência ou excesso a campo.

\section{Considerações finais}

O N aplicado em cobertura no cultivo de girassol sob sistema de plantio direto aumenta $\mathrm{O}$ seu teor nas folhas, mas não altera os teores de $\mathrm{P}$, $\mathrm{K}, \mathrm{Ca}, \mathrm{Mg}, \mathrm{Fe}, \mathrm{Mn}$, Cu e $\mathrm{Zn}$.

\section{Referências}

BAYER, C.; SCHENEIDER, N. G. Plantio direto e o aumento no conteúdo de matéria orgânica do solo em pequenas propriedades rurais no município de Teutônia. Ciência Rural, Santa Maria, v. 29, n.1, p.155-166,1999.

BORKERT, C. M. et al. Efeito residual da adubação potássica, sobre girassol e milho, em três diferentes latossolos roxos. Pesquisa Agropecuária Brasileira, Brasília, v. 32, n. 12, p. 1227-1234, 1997.

CARTER, J. F. Sunflower science and tecnology. Madison: American Society of Agronomy, 1978. p. 505. (Series Agronomy, v. 19).

CASTIGLIONI, V. B. R. et al. Fases de desenvolvimento da planta de girassol. Londrina: EMBRAPA: CNPSo, 1994. (Documentos, 58). p. 24.

CASTRO, C. de. et al. Cultura do girassol. Circular Técnica, 13. Londrina: EMBRAPA: CNPSo, 1997. p. 36.

DEIBERT, E. J.; UTTER, R. A. Sunflower Growth and Nutrient Uptake: Response to Tillage System, Hybrid Maturity and Weed Control Method. Soil Sci. Soc. Am. J. v. 53, p. 133-138. 1989.

EMBRAPA/CNPS. Sistema Brasileiro de Classificação de Solos. São Paulo,SP: EMBRAPA,SPI, 1999. p. 412.

EMBRAPA/CNPSO. Informes da avaliação de genótipos de girassol, 1996/97 e 1997. Londrina: EMBRAPA : CNPSo, 1997. p. 116. (Documentos, 110).

FUNDAÇÃO Instituto Agronômico Do Paraná. Cartas climáticas do Estado do Paraná. Londrina, 1984. p. 45.

HILDEBRAND, C. Manual de análise química de solos e plantas. Curitiba: UFPR. 1977. p. 254.

KOELHER, H. S. Estatística experimental. Curitiba: Universidade Federal do Paraná, 1998. p. 124.

LEITE, R. M. V. B. C. Doenças do girassol. Circular Técnica, 19. Londrina: EMBRAPA: CNPSo, , 1997. p. 68.

LARCHER, W. Ecofisiologia Vegetal. São Carlos: RiMa, 2000. p. 531. 
MACHADO, P. R. Absorção de nutrientes por duas cultivares de girassol (Helia nthus annuus L) em função da idade e adubação, em condições de campo. 1979. 83f. Dissertação (Mestrado) - Piracicaba: ESALQ. 1979.

MALAVOlTA, E.; VITTI, G. C.; OlIVEIRA, S. A. Avaliação do estado nutricional das plantas: princípios e aplicações. 2. ed. Piracicaba: Potafós, 1997. p. 319.

MARSCHNER, H. Mineral Nutrition of Higher Plants. $5^{\text {th }}$. San Diego: Academic, 1993.

MENGEL, K.; KIRKBY, E. A. Principles of plant nutrition. $4 .^{\text {th }}$. Bern: International Potash Institute, 1987. p. 687.

MUZILLI, O. Manejo da fertilidade do Solo. In___. Fundação Instituto Agronômico do Paraná. Plantio direto no Estado do Paraná. Circular, 23. Londrina: IAPAR. 1981. p.43-58.

MUZILLI, O. Fertilidade do solo em plantio direto. In__ . FANCELLI, A. L. Atualização em plantio direto. Campinas: Fundação Cargil, 1985. p.147-160.

ROSSI, R. O. Adubação em girassol. São Miguel do Oeste: Santa Catarina, 1991. p. 31.

ROSSI, R. O. Girassol. Curitiba: Tecnoagro, 1998. p. 333.

SALISBURY, F. B.; ROSS, C. W. Plant Physiology. $4^{\text {th }}$ ed. California: Wadsworth, 1992. p. 682.
SAMENI, A. M. et al. Effect of fertilizer-N and herbicides on the growth and $\mathrm{N}$ content of sunflower. Agronomy Journal, Madison, v. 68, p. 285-288, 1976.

SCHNEITER, A. A.; MILLER, J. F. Description of sunflower grow stages. Crop Science, v. 6, n. 21, p. 901-903, 1981.

SFREDO, G. J.; CAMPO, R. J.; SARRUGE, J. R. Girassol: nutrição mineral e adubação. Circular técnica, 8. Londrina: EMBRAPA: CNPSo, 1984. p. 36.

SFREDO, G. J.; SARRUGE, J. R. Acúmulo de micronutrientes em plantas de girassol. Pesquisa Agropecuária Brasileira, Brasília, v. 25, n. 4, p. 499-503, 1990.

SFREDO, G. J.; SARRUGE, J. R. Concentração de micronutrientes em órgãos de plantas de girassol. Pesquisa Agropecuária Brasileira, Brasília, v. 25, n. 12, p. 1727-1732, 1990.

TOMÉ, J. B. Jr. Manual para interpretação de análise de solo. Guaíba: Agropecuária, 1997. p. 247.

VRÂNCEANU, A. V. El Girassol. Madrid: Mundi, 1977. p. 379. 